R.M. Harrison, R.E. van Grieken (eds)

\section{Atmospheric Particles}

Wiley, Chichester 1998

610 pp.; $£ 110.00$

ISBN 0-471-95935-9

This book is the fifth volume in the IUPAC series on 'Analytical and Physical Chemistry of Environmental Systems' (Series editors: J. Buffle and H.P. van Leeuwen).

A title as broad as 'Atmospheric Particles' indicates that the editors have embarked on a huge task. Nevertheless, this is a substantial work and provides depth as well as breadth for this all-important subject. The considerable and rapidly growing literature on particulates in the atmosphere has been stimulated in part by the perceived detriment to human health in susceptible groups in the population who inhale these particles. This volume is a multi-author work with a preponderance of European authors. It covers the key areas of the physical as well as chemical characteristics of atmospheric particles, how they are formed and how they behave.

Because particle size is always a vital attribute of any aerosol, it is appropriate that the first chapter deals with size distributions. This treatment is not self-explanatory everywhere, and calls for some prior knowledge of the mathematical basis of particle size analysis. Vertical and horizontal (geographic) variations are discussed. This introduction dovetails nicely into an excellent extended chapter on aerosol sampling. Covering both theory and practice, the wide range of different types of sampling devices is clearly and critically explained. Methods for the measurement of particle size are then described, also measurements of particle deposition on the ground (which is also the topic of an entire subsequent chapter on 'dry deposition' that is itself extensive and deals with both modelling and experimental work); the account finishes with a discussion of sampling strategies in relation to scientific and practical requirements.

The chemical composition of atmospheric particles is treated in several chapters which form the backbone of the book. These are all well documented and presented, and together provide a valuable source of reference. They cover inorganic composition, speciation, micro-analysis as applied to individual particles, trace metals, the carbonaceous component of combustion aerosols and the association of atmospheric particles with dioxins, PCBs and polycyclic aromatic hydrocarbons. Other chapters cover atmospheric particles of biological origin, ranging in size from virus particles to pollen grains and the role of biogenic gases such as dimethyl sulphide and ammonia in secondary aerosol generation. There is also a chapter on kinetics and the thermodynamic properties of aqueous aerosols.

The book concludes with three chapters on atmospheric phenomena per se: one covers 'wet processes' involving atmospheric particles, i.e. the effects of water vapour on particle formation, on growth by condensation and on scavenging by precipitation; then there is a separate account of hazes, fog and clouds, and finally, a discussion on the impact of atmospheric particles on the global environment.

This book will be of interest to a wide readership, especially anyone with a physics and/or chemistry background who wishes to survey the whole topic of the properties and behaviour of aerosols in the atmosphere. More importantly, it will be a useful source of reference for many interested in these and related aspects of the study of particles in the atmosphere.

G. Patrick, Leicester

\section{Colin J. Sanderson (ed.) \\ Interleukin-5 from Molecule to Drug Target for Asthma}

Lung Biology in Health and Disease, vol. 125

Executive editor Claude Lenfant

Dekker, New York 1998

410 pp.; $\$ 185.00$

ISBN 0-8247-0190-9

Interleukin-5 (IL-5) was first discovered in 1983 by Swain et al. as a B cell growth factor, which promoted the proliferation and production of IgM by the murine B cell tumour line Bcl-1 and was not cloned until 1986 by Honjo et al. Over the 14 years since that time, IL-5 has emerged as perhaps one of the key regulators of eosinophil production and function and is therefore an important molecule implicated in the pathogenesis of allergic diseases. IL-5 induces the differentiation and expansion of eosinophil precursors in the bone marrow and plays a key role in regulating many of the specialised functions of mature terminally differentiated eosinophils, such as chemotaxis, activation and enhancing their survival at allergic tissue sites. This clear link between IL-5 and eosinophils, first described by Sanderson's group, has been the basis for a significant investment from industry to find new agents that will block IL-5 and control the production of eosinophils and thereby the clinical expression of asthma and allergic diseases. The validity of IL- 5 as a target has been demonstrated in a variety of animal models of allergic asthma, where administration of neutralising anti-IL-5 monoclonal antibodies in mouse, guinea pigs or primate models of allergic asthma have demonstrated inhibition in the development of airway eosinophilia. This has been further supported by the observation that mice, rendered IL-5 deficient through deletion of the IL-5 gene, fail to produce either blood or lung eosinophilia in response to aeroallergen challenge. How these observations translate to its use as a therapeutic drug in the management of asthma and eosinophilic diseases in humans remains to be determined.

\section{KARGER}

(C) 1999 S. Karger AG, Basel

Fax + 41613061234

E-Mail karger@karger.ch

www. karger.com
Accessible online at:

http://BioMedNet.com/karger 
Nevertheless, this viewpoint has driven research into all aspects of IL-5 at several distinguished laboratories over the world and has led to a tremendous increase in our knowledge and understanding of the biology of this crucial molecule. Although there have been several excellent reviews on IL-5, there has been no thorough and complete overview. 'Interleukin-5 from molecule to drug target for asthma' edited by Colin Sanderson is the first such excellent overview on IL-5 published so far, and especially, comes at a time when new anti-IL-5 therapeutics are being developed and tested. This important book contains 18 chapters covering all aspects of IL-5 from clinical and experimental research and molecular biology to insights into new drug discovery programs, pooling the insights of over 65 biological researchers, pharmaceutical scientists and clinicians.

The book has exemplary coverage of developments occurring in all the fields of IL-5 studies. It highlights the structure and function of IL-5 and its receptor, its signal transduction pathways and explores the interaction of IL-5 with eotaxin. It also outlines the regulation of IL-5 gene expression and analyses agents that down-regulate IL-5 production, evaluates studies on IL-5 knockout and transgenic mice and discusses the role of monoclonal antibodies directed against IL-5 in animal models of asthma. The book ends with an interesting chapter on the pulmonary biology of anti IL-5 antibodies. However, like all major cytokines discovered to date, it is the pathological arm of the molecule that is always overemphasised, and one then tends to get the impression that the only role of IL-5 is to produce asthma and allergy. Certainly, nature must have produced this molecule not with an intention of producing disease, but to perform important physiological functions. Unfortunately, this physiological side of the molecule has not been covered in the book. Although the major effect of IL-5 is thought to be on the eosinophil lineage, since this is the most studied function of this molecule, recent studies with IL-5 deficient animals have demonstrated an important role for IL-5 on B cell development, in particular, on IgA production. While developing strategies targeted at blocking IL-5 to treat allergic disease states, one needs to be aware that this could have profound effects on the physiological role performed by IL-5, which have largely been underresearched and neglected.

Nevertheless, this is an excellent book for those working in research into asthma and allergic diseases. It will be an important reading for students and clinicians interested in the molecules behind allergy and asthma, for pharmaceutical chemists developing new anti-asthma drugs, and also for cytokine biologists working on protein structure and gene regulation and immunologists interested in the remarkable diversity of the immune response to allergic reactions. I would strongly recommend this book to everyone interested in the quest for knowledge; especially those interested in understanding the role of different molecules in asthma and allergic diseases.

Sundeep Salvi, Southampton 